\title{
Dual Extended Kalman Filter for the Identification of Time-Varying Human Manual Control Behavior
}

\author{
Alexandru Popovici* \\ San Jose State University, NASA Ames Research Center \\ Peter M. T. Zaal ${ }^{\dagger}$ \\ Daan M. Pool ${ }^{\ddagger}$ \\ San Jose State University, NASA Ames Research Center \\ Delft University of Technology
}

\begin{abstract}
A Dual Extended Kalman Filter was implemented for the identification of time-varying human manual control behavior. Two filters that run concurrently were used, a state filter that estimates the equalization dynamics, and a parameter filter that estimates the neuromuscular parameters and time delay. Time-varying parameters were modeled as a random walk. The filter successfully estimated time-varying human control behavior in both simulated and experimental data. Simple guidelines are proposed for the tuning of the process and measurement covariance matrices and the initial parameter estimates. The tuning was performed on simulation data, and when applied on experimental data, only an increase in measurement process noise power was required in order for the filter to converge and estimate all parameters. A sensitivity analysis to initial parameter estimates showed that the filter is more sensitive to poor initial choices of neuromuscular parameters than equalization parameters, and bad choices for initial parameters can result in divergence, slow convergence, or parameter estimates that do not have a real physical interpretation. The promising results when applied to experimental data, together with its simple tuning and low dimension of the state-space, make the use of the Dual Extended Kalman Filter a viable option for identifying time-varying human control parameters in manual tracking tasks, which could be used in real-time human state monitoring and adaptive human-vehicle haptic interfaces.
\end{abstract}

\section{Nomenclature}

$\begin{array}{llll}e & \text { error signal, }- & n & \text { pilot remnant } \\ F & \text { derivative of the state equation } & P & \text { state covariance matrices } \\ f & \text { state equation } & Q & \text { process noise covariance matrix } \\ f_{f} & \text { forcing function, }- & q^{2} & \text { error signal variance factor } \\ G & \text { derivative of the output equation } & R & \text { measurement noise variance } \\ g & \text { output equation } & r^{2} & \text { control signal variance factor } \\ H_{c} & \text { controlled dynamics } & s & \text { Laplace operator } \\ H_{p} & \text { human controller response } & T_{L} & \text { pilot lead time constant, s } \\ I & \text { identity matrix } & t & \text { time, s } \\ K & \text { Kalman gains } & u & \text { pilot control input, }- \\ K_{p} & \text { visual position gain, }- & \hat{u} & \text { modeled control input, }- \\ K_{v} & \text { visual rate gain, }- & x_{s} & \text { state filter state vector }\end{array}$

\footnotetext{
*Research Associate, Human Systems Integration Division, NASA Ames Research Center, Moffett Field, CA, 94035; alexandru.popovici@nasa.gov. Member.

${ }^{\dagger}$ Senior Research Engineer, Human Systems Integration Division, NASA Ames Research Center, Moffett Field, CA, 94035; peter.m.t.zaal@nasa.gov. Member.

${ }^{\ddagger}$ Associate Professor, Faculty of Aerospace Engineering, Delft University of Technology, Delft, The Netherlands; d.m.pool@tudelft.nl. Member.
} 


\begin{tabular}{|c|c|c|c|}
\hline$\theta$ & parameter filter state vector & \multicolumn{2}{|c|}{ Subscripts } \\
\hline$\zeta_{n}$ & neuromuscular damping, - & & \\
\hline$\sigma$ & standard deviation & 0 & initial value \\
\hline$\tau_{v}$ & visual time delay, s & $k$ & discrete time index \\
\hline$\Phi$ & discrete version of $F$ & $p$ & parameter filter \\
\hline$\omega_{n}$ & neuromuscular frequency, rad s ${ }^{-1}$ & $s$ & state filter \\
\hline$w$ & process noise & \multirow{2}{*}{\multicolumn{2}{|c|}{ Abbreviations }} \\
\hline$v$ & measurement noise & & \\
\hline$y$ & controlled dynamics output & $\operatorname{cov}$ & covariance \\
\hline$\Gamma$ & discrete input identity matrices & diag & diagonal matrix \\
\hline$\Delta t$ & time step, s & DEKF & Dual Extended Kalman Filter \\
\hline \multicolumn{2}{|c|}{ Superscripts } & $\begin{array}{l}\text { MLE } \\
\text { VAF }\end{array}$ & $\begin{array}{l}\text { Maximum Likelihood Estimation } \\
\text { variance accounted for }\end{array}$ \\
\hline- & prediction & & \\
\hline+ & correction & & \\
\hline & total & & \\
\hline
\end{tabular}

\section{Introduction}

In manual control tasks, humans combine the perception of different cues from the outside environment in order to perform suitable control actions. Linear, time-invariant transfer functions have been widely used to model human manual control behavior and can explain a large part of the mechanism behind it! 1 These models capture equalization dynamics, required for a stable closed-loop performance, neuromuscular limitations, and a stochastic signal, the remnant, that captures the part of the human's control input that is not explained by the linear models.

In reality, however, there are many scenarios in which human control behavior is highly time-varying. First, humans adapt their control behavior depending on vehicle dynamics ${ }^{1}$ For example, changing external conditions, systems failure, or flying close to the stall regime, can result in sudden changes vehicle dynamics that affect human control strategy. Second, intrinsic behavioral factors such as fatigue, motivation and stress can also result the human operator to adopt different manual control strategies. Identifying when these changes occur and understanding how human behavior adapts to these changes, could be used in the design process of haptic control and failure detection systems, and potentially as a tool for real-time monitoring of human state and performance. Thus, the need for a real-time, time-varying human control parameter identification technique is evident.

Multiple methods exist currently for the estimation of human control model parameters. Frequency domain techniques or linear time-invariant models were successfully used in the past to obtain human control models. ${ }^{[2] 3}$ For these methods to provide parameter estimates with a small bias, a high signal-to-noise ratio is required, which can only be obtained by averaging time-domain signals in order to reduce the effect of the stochastic remnant signal. This, however, imposes a severe limitation on the real-time identification of parameters. Furthermore, special considerations are required for the design of forcing functions used in the experiment. A time-domain identification technique based on Maximum Likelihood Estimation has also been successfully applied for the identification of multichannel, pilot models $\frac{4}{4}$ The same technique was used to identify time-varying human control behavior, $\frac{5}{5}$ however a scheduling function for the parameter variation was required in advance. This limits the use of the technique for real-world applications, where no prior knowledge is available on the changes of the parameters. Wavelet transforms were also used for the identification of time-varying human control frequency response functions, however this technique does not generally allow for the direct estimation of the time-varying human control model parameters ${ }^{6}$

Kalman filters ${ }^{7}$ have been widely used for the estimation of both the states and the parameters. There are typically two approaches by which this can be achieved. In the joint estimation, the parameters are included in an augmented state space model and the regular state estimation is performed $\underline{8}$ In this estimation approach, the multiplication between the states and the parameters makes the system inherently nonlinear. Therefore, the Extended Kalman Filter or the Unscented Kalman Filter methods are typically used. In the dual estimation technique, which is suggested to have better convergence properties, two separate filters run concurrently: one that estimates the states of a model, and one that estimates the parameters ${ }^{9}$ This allows 
the separation of the nonlinear system into two separate, less strongly nonlinear estimation problems. The results from the state filter are used in the parameter filter and vice versa 10 These methods have been used in several studies for the estimation of both the states and the parameters of electrical and mechanical systems. ${ }^{11}[12$ Schiess et al. attempted the estimation of human control model parameters using an Exteded Kalman Filter, in which all the parameters were augmented in a single the state-space representation.13 While the filter manages to reasonably estimate the parameters on computer generated data, it mostly diverges when applied to experimental data. With careful choice of the parameter process noise covariances, the filter managed to converge, but the estimation of all parameters was rarely achieved.

The goal of this paper is to develop a Dual Extended Kalman Filter (DEKF) implementation aimed at the identification of time-varying, single-axis human control model parameters. The performance of the filter is analyzed on simulated data, as well as experimental data obtained from a previous experimental study ${ }^{[}$

\section{Human Control Model}

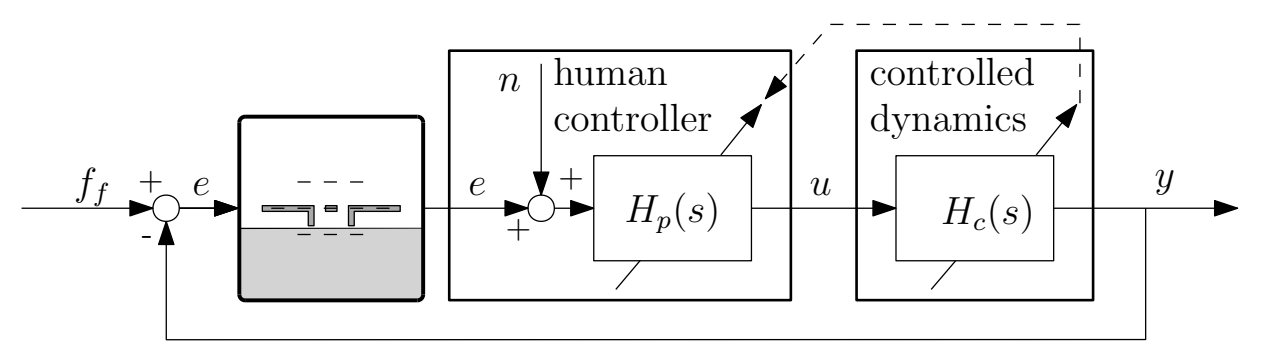

Figure 1: Compensatory tracking task control diagram.

The control diagram of a typical single-axis manual tracking task is depicted in Figure 1) The goal of the human operator is to continuously minimize the error $e$ presented on the simplified primary flight display (PFD), by providing control inputs $u$ with a joystick. These inputs are transformed into the output $y$ through the controlled dynamics transfer function $H_{c}$. The error $e$ represents the difference between the target forcing function $f_{f}$ and the output $y$.

In such tasks, human control behavior can be represented by a quasi-linear human operator model that is composed of two parts ${ }^{1} \mathrm{~A}$ linear transfer function, $H_{p}$ captures equalization dynamics, time delays, and neuromuscular limitations. The second part is represented by a remnant signal $n$, which accounts for the human controller's nonlinear behavior. This signal is modeled as a first-order low pass filter according to Levison's remnant model, $\stackrel{14}{14}$ which makes this signal inherently colored.

According to McRuer's crossover model, humans adjust their control strategy such that the human controller-vehicle dynamics open-loop transfer function approximates a single integrator near the crossover frequency 11 If the controlled dynamics are time-varying, then the human controller likely also becomes timevarying, achieving the required performance and stability requirements. Considering controlled dynamics close to a double-integrator, significant visual lead is required. The human operator transfer function $H_{p}$ then becomes:

$$
H_{p}(s)=K_{p}\left(1+T_{L} s\right) e^{-\tau_{v} s} \frac{\omega_{n}^{2}}{\omega_{n}^{2}+2 \zeta_{n} \omega_{n} s+s^{2}}=\left(K_{p}+K_{v} s\right) e^{-\tau_{v} s} \frac{\omega_{n}^{2}}{\omega_{n}^{2}+2 \zeta_{n} \omega_{n} s+s^{2}}
$$

Visual gain $K_{p}$ and visual lead time constant $T_{L}$ represent the human operator's equalization used to achieve the desired performance, whereas physical limitations are given by the visual time delay $\tau_{v}$, neuromuscular damping $\zeta_{n}$ and neuromuscular frequency $\omega_{n}$. The equation can be rewritten such that the equalization is represented by two gains, one on the error position signal, $K_{p}$, and one on the error velocity signal, $K_{v}$. Assuming that the control input excites the system sufficiently, there are five identifiable parameters from Equation (11): $K_{p}, K_{v}, \tau_{v}, \omega_{n}$, and $\zeta_{n}$.

\section{Dual Extended Kalman Filter}

As mentioned in Section \. a dual Kalman filter implementation uses two separated filters, one that estimates the states of the system and one that estimates the parameters of interest. Applied to the human 
manual control identification problem, two concurrent filters are used, one estimating the states and equalization parameters of the human controller model, which will be called the state filter throughout this paper, and one that estimates the time delay and neuromuscular parameters, which will be called the parameter filter. A diagram of the two filters working in parallel is shown in Figure 2

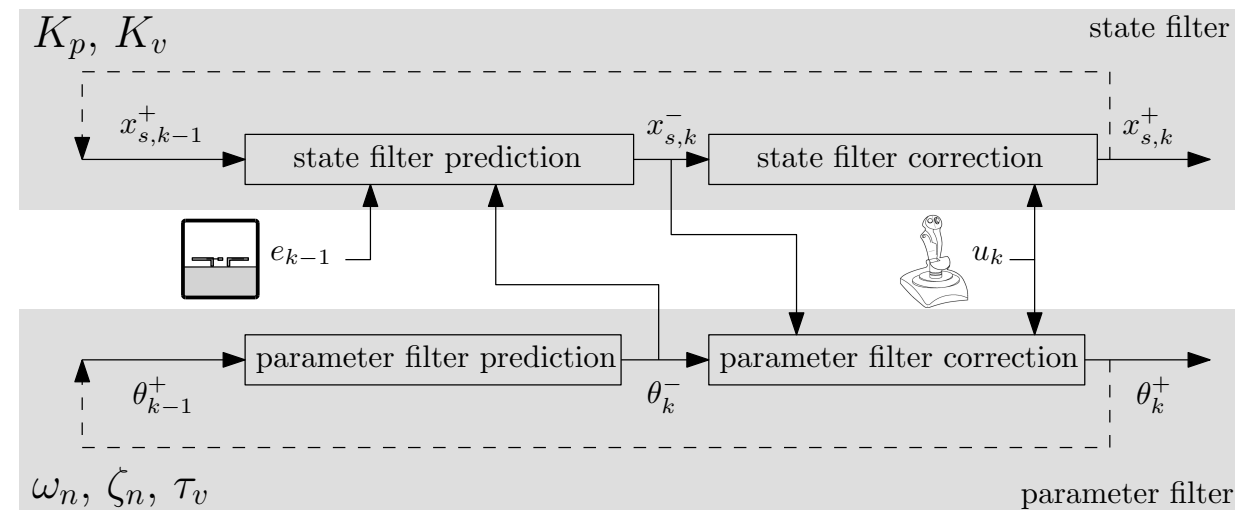

Figure 2: The state and parameter filters.

The parameter filter prediction $\theta_{k}^{-}$is used in the state filter prediction $x_{s, k}^{-}$, and the prediction of the state filter is used in the parameter filter correction, $\theta_{k}^{+}$. The error signal $e$ only drives the state filter prediction, as an external input. The control input $u$ is used in the measurement equation of both filters. A detailed representation of all system equations for both filters is given in Appendix A.

\section{A. Filter implementation}

For the implementation of the Kalman filters, a state-space representation of the model is required. In order to convert the time delay $\tau_{v}$ into a state-space representation, a $3^{\text {rd }}$ order Padé approximation is used, making the need for the Extended version of the filters apparent.

The state-space representation of the state filter is given in Equations (2) and (3):

$$
\begin{aligned}
\dot{\boldsymbol{x}}_{\boldsymbol{s}}(t) & =f\left(\boldsymbol{x}_{\boldsymbol{s}}(t), e(t), \boldsymbol{\theta}(t)\right)+\boldsymbol{w}_{\boldsymbol{s}}(t) & & \operatorname{cov}\left(\boldsymbol{w}_{\boldsymbol{s}}\right)=Q_{s} \\
u(t) & =g\left(\boldsymbol{x}_{\boldsymbol{s}}(t), \boldsymbol{\theta}(t)\right)+v(t) & & \operatorname{cov}(v(t))=R
\end{aligned}
$$

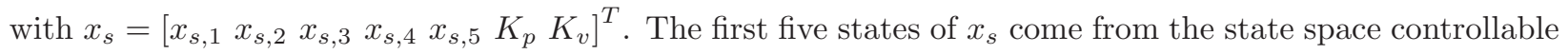
canonical form, and do not have real physical meaning. Error position and error velocity parameters are augmented in the state vector of the state filter, since they only appear in the output equation $g$ (see Equations (40) and (41)). $\boldsymbol{\theta}(t)$ is the parameter vector estimated by the second filter, $e(t)$ and $u(t)$ the error and control input signals as indicated in Figure 1, Zero mean, Gaussian process and measurement noises are captured in the $w_{s}(t)$ and $v(t)$ signals. The process noise covariance $Q_{s}$ is $7 \times 7$ diagonal matrix, and $R$ represents the variance of the measurement noise of the only output signal $u(t)$. The parameter filter state space equations are shown in Equations (4) and (5):

$$
\begin{array}{ll}
\dot{\boldsymbol{\theta}}(t)=\boldsymbol{w}_{\boldsymbol{p}}(t) & \operatorname{cov}\left(\boldsymbol{w}_{\boldsymbol{p}}(t)\right)=Q_{p} \\
u(t)=g\left(\boldsymbol{x}_{\boldsymbol{s}}(t), \boldsymbol{\theta}(t)\right)+v(t) & \operatorname{cov}(v(t))=R
\end{array}
$$

with $\theta=\left[\omega_{n} \zeta_{n} \tau_{v}\right]^{T}$. The measurement equation is the same as for the state filter. It can be seen that the evolution of the time-varying parameter vector $\theta$ is only driven by the zero mean white process noise $w_{p}$. The variance of $w_{p}$ is given by the diagonal elements of the $3 \times 3$ matrix $Q_{p}$, the process noise covariance matrix. Modeling unknown time-varying parameters as a random walk process proved to be a successfull and general method in previous research $\frac{15}{15}$ The same method is applied for the states $K_{p}$ and $K_{v}$ in the state filter.

The discrete time equations of the two filters running in parallel are given below. 
Parameter filter prediction:

$$
\begin{aligned}
& \boldsymbol{\theta}_{k}^{-}=\boldsymbol{\theta}_{k-1}^{+} \\
& P_{p, k}^{-}=\Phi_{p, k-1} P_{p, k-1}^{+} \Phi_{p, k-1}^{T}+\Gamma_{p, k-1} Q_{p} \Gamma_{p, k-1}^{T}
\end{aligned}
$$

State filter prediction:

$$
\begin{aligned}
& \boldsymbol{x}_{s, k}^{-}=\boldsymbol{x}_{s, k-1}^{+}+f\left(\boldsymbol{x}_{s, k-1}^{+}, e_{k-1}, \theta_{k}^{-}\right) \Delta t \\
& P_{s, k}^{-}=\Phi_{s, k-1} P_{s, k-1}^{+} \Phi_{s, k-1}^{T}+\Gamma_{s, k-1} Q_{s} \Gamma_{s, k-1}^{T}
\end{aligned}
$$

State filter (partial) correction:

$$
\begin{aligned}
& K_{s, k}=P_{s, k}^{-} G_{s, k}^{T}\left[G_{s, k} P_{s, k}^{-} G_{s, k}^{T}+R\right]^{-1} \\
& P_{s, k}^{+}=\left(I-K_{s, k} G_{s, k}\right) P_{s, k}^{-}\left(I-K_{s, k} G_{s, k}\right)^{T}+K_{s, k} R K_{s, k}^{T}
\end{aligned}
$$

Parameter filter correction:

$$
\begin{aligned}
& K_{p, k}=P_{p, k}^{-}\left(G_{p, k}^{t o t}\right)^{T}\left[G_{p, k}^{t o t} P_{p, k}^{-}\left(G_{p, k}^{t o t}\right)^{T}+R\right]^{-1} \\
& \boldsymbol{\theta}_{k}^{+}=\boldsymbol{\theta}_{k}^{-}+K_{p, k}\left[u_{k}-g\left(\boldsymbol{x}_{s, k}^{-}, e_{k}, \boldsymbol{\theta}_{k}^{-}\right)\right] \\
& P_{p, k}^{+}=\left(I-K_{p, k} G_{p, k}^{t o t}\right) P_{p, k}^{-}\left(I-K_{p, k} G_{p, k}^{t o t}\right)^{T}+K_{p, k} R K_{p, k}^{T}
\end{aligned}
$$

Final state filter correction:

$$
\boldsymbol{x}_{s, k}^{+}=\boldsymbol{x}_{s, k}^{-}+K_{s, k}\left[u_{k}-g\left(\boldsymbol{x}_{s, k}^{-}, e_{k}, \boldsymbol{\theta}_{k}^{-}\right)\right]
$$

Where :

$k$, the discrete time index

$\Delta t$, the time step

$\Phi_{s, k-1}$, the discrete version of $F_{s}=\left.\frac{\partial f\left(\boldsymbol{x}_{s}, e_{k-1}, \boldsymbol{\theta}_{k}^{-}\right)}{\partial \boldsymbol{x}_{s}}\right|_{\boldsymbol{x}_{s}=\boldsymbol{x}_{s, k-1}^{+}}$

$\Gamma_{\{s, p\}, k-1}$, the discrete input identity matrices of the state and parameter filters

$G_{s, k}=\left.\frac{\partial g\left(\boldsymbol{x}_{s, k}, \boldsymbol{\theta}_{k}^{-}\right)}{\partial \boldsymbol{x}_{s, k}}\right|_{\boldsymbol{x}_{s, k}=\boldsymbol{x}_{s, k}^{-}}$

$G_{p, k}^{t o t}=\left.\frac{d g\left(\boldsymbol{x}_{s, k}^{-}, \boldsymbol{\theta}\right)}{d \boldsymbol{\theta}}\right|_{\boldsymbol{\theta}=\boldsymbol{\theta}_{k}^{-}}$

$K_{\{s, p\}, k}$, the Kalman gains

$P_{\{s, p\}, k}$, the state covariance matrices

Note that, since not all the elements of the parameter filter $\theta$ appear in the output function $g$ as presented in Appendix $\mathrm{A}$, and the fact that the filters depend on each other, the numerical calculation of the total derivative of the output function with respect to the states of the parameter filter is necessary, as indicated by $\frac{d g\left(\boldsymbol{x}_{s, k}, \boldsymbol{\theta}\right)}{d \boldsymbol{\theta}}$. The steps required to compute this derivative, together with a more detailed representation of the equations above, are presented in Appendix $\mathrm{A}$

\section{B. Considerations}

\section{Remnant}

The noise in the control loop is captured in the remnant signal. Since the control task is closed loop system, the remnant can be seen as affecting both the process and measurement noises. Levison et al! 16 showed that 
the remnant can be modeled as a first-order low-pass filter injected in the error signal (human controller's input), making the remnant an inherently colored noise. The process and measurement noise need to be white, otherwise the Kalman filter is not optimal. In order to overcome this issue, the remnant can be included in the state filter as a first order low pass filter driven by white noise. However, as Levison found, the parameters of such filter change depending on the order of the controlled dynamics, as well as the ratio between error velocity and error position variances. Since these parameters are not known a priori, it becomes difficult to have an accurate representation of the remnant parameters. In this study, it was decided not to account for the remnant in the state filter in order to avoid additional uncertainty, and assume that its contribution is part white process noise and part white measurement noise.

\section{Measurement noises}

Measurement noise is typically associated with errors in the sensors used to measure system outputs, whereas process noise typically refers to errors in the modeling of a certain system. In the case of the human control model, the uncertainty of the model is difficult to quantify due to the inherent variability of human control behavior. Moreover, there is no "sensor noise". Therefore, in this study, the covariance matrices $Q_{s}, Q_{p}$ and $R$ are parameters that are tuned to achieve parameter estimation performance, and have no real physical interpretation.

Many experiments that looked at identification of human manual control parameters found that the power of the remnant accounts for $20-30 \%$ of the power of the control input $u$ in Figure 1. In this study the measurement noise covariance $R$ described in Equation (3) is considered time varying, and is modeled as:

$$
R(k)=r^{2} \sigma_{u(k-5 / \Delta t: k)}^{2}
$$

meaning that the measurement noise power at time step $k$ is a factor $r^{2}$ of the variance of the control input from the last five seconds before time step $k$. For example, in the case where the controlled dynamics $H_{c}$ change from single integrator to double integrator, the humans typically increase the control input power, therefore there will be more noise in the control loop which has to be accounted for by the two filters. A value of of 4 for the $r^{2}$ factor showed good filter performance for the experimental data in Section IV] and a value of 0.7 was chosen when the DEKF was applied on simulation data. A possible reason for the need of a higher measurement noise power with the experimental data is the presence of more noise sources that exist in a real scenario but not in the simulations, such as higher noise from the control input device, nonlinear human control behavior, etc.

\section{Process noise}

There are two process noise covariance matrices, one for the state filter and one for the parameter filter. The process noise covariance matrix is given by:

$$
Q_{s}(k)=\operatorname{diag}\left(\left[\begin{array}{lllllll}
0 & 0 & 0 & 0 & q^{2} \sigma_{e(k-5 / \Delta t: k)}^{2} & K_{p, 0} K_{v, 0}
\end{array}\right]\right)
$$

where $K_{p, 0}$ and $0.1 K_{v, 0}$ are the initial conditions for the position gain and velocity gain, and $q^{2}$ represents a proportion of the error signal variance. Note that the $5^{t h}$ state of the state filter relates to the error signal $e$. This accounts for the fact that humans will not perfectly follow the linear model while controlling, and can be seen as system error, which is difficult to know in advance. A value of 0.4 for $q^{2}$ is used for both the simulation and experimental data presented in Section IV] The constant process noise covariance for the parameter filter is given by:

$$
Q_{p}=\operatorname{diag}\left(\left[\begin{array}{lll}
0.1 \omega_{n, 0} & 0.1 \zeta_{n, 0} & 0.1 \tau_{v, 0}
\end{array}\right]\right)
$$

where again, $\omega_{n, 0}, \zeta_{n, 0}$ and $\tau_{v, 0}$ are the initial parameters from the parameter filter.

As a rule of thumb, for the state filter it was found that the setting the last two diagonal elements of the process noise covariance matrix equal to $100 \%$ of the initial values of $K_{p}$ and $K_{v}$ results in good filter

performance, for the measurement noise presented above. For the parameter filter, values of $10 \%$ of the initial parameter estimates were used for the diagonal elements of $Q_{p}$. 
This choice for the process noise covariance matrix of the parameters represents how large their variation is expected to be over time. Since the equalization dynamics $\left(K_{p}, K_{v}\right)$ are expected to change a lot more than neuromuscular parameters and time delay $\left(\omega_{n}, \zeta_{n}, \tau_{v}\right)$ during changes in controlled dynamics $H_{c}$, they have higher process noise covariance values. However, a larger (or smaller) choice of measurement noise power will have to be accounted for in the process noise by increasing (or decreasing) its covariance.

Note that since the measurement noises does not have a clear physical interpretation, similar filter performance can be achieved with different choices of the process noise and measurement noise combinations.

This representation of the process and measurement noise covariances, together with the initial conditions, are aimed as a guideline to facilitate the tuning of the filter. The two most important parameters for convergence are therefore the factors $r^{2}$ and $q^{2}$, together with the initial covariance matrices $P_{\{s, p\}, 0}$, which depend on how far (or close) the initial parameter guesses are from the true parameters.

\section{Initialization}

Initial parameter estimates are important for the performance of the filter. With the Extended Kalman filter, a poor choice of the initial estimates can result in filter divergence. Furthermore, even if the filters do not diverge, convergence may be slow if the initial state covariance matrices do not account for the initial state estimates being far from the true values $\frac{17] 18}{18}$

The method based on Maximum Likelihood Estimation presented in [5] is used to obtain initial estimates of the parameters of interest. Although the estimation will be biased in the presence of high remnant power, the initial estimates obtained are sufficiently accurate to initialize the DEKF with.

In case the MLE method is not used, generic values for the initial parameters can be applied as follows:

$$
\begin{aligned}
K_{p, 0} & =\sigma_{u} / \sigma_{e} \\
K_{v, 0} & =0.5 K_{p, 0} \\
\omega_{n, 0} & =10 \mathrm{rad} / \mathrm{s} \\
\zeta_{n, 0} & =0.3 \\
\tau_{v, 0} & =0.3 \mathrm{~s}
\end{aligned}
$$

The initial error position gain $K_{p}$ can be approximated by the ratio of the standard deviation of the control input and error signals, respectively. Furthermore, controlled dynamics that are between a single and double integrator have a lead time constant equal to the break frequency of the controlled dynamics $H_{c}$. A value of 0.5 was used in this case, resulting in the estimated $K_{v}$. The other states in the state filter have initial conditions equal to zero. The values chosen for $\omega_{n, 0}, \zeta_{n, 0}$ and $\tau_{v, 0}$ are typically obtained for human control parameters in compensatory tracking tasks.

The initial state covariance matrix for the state filter was chosen as:

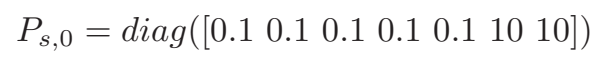

and for the parameter filter:

$$
P_{p, 0}=\operatorname{diag}\left(\left[\begin{array}{lll}
10 & 1 & 1
\end{array}\right]\right)
$$

For the measurement noise of the state and parameter filters, the initial value was chosen to be:

$$
R_{0}=r^{2} \sigma_{u, 5}^{2}
$$

where $\sigma_{u, 5}^{2}$ represents the variance of the control input over the first 5 seconds. $r^{2}$ has the same value as in Equation (24). 


\section{Preliminary results}

\section{A. Simulation}

A Simulink closed-loop simulation was created, in which the human controller model parameters were either constant, or they all varied according to sigmoid functions. The remnant was modeled according to Levison's model, injected into the error signal as shown in Figure 1.

\section{Constant parameters}

A 90 second closed-loop simulation sampled at $100 \mathrm{~Hz}$ s was performed, where the controlled dynamics and pilot model parameters were kept constant. The remnant had a break frequency of $3 \mathrm{rad} / \mathrm{s}$, and the gain was adjusted such that the remnant power at the control input location has a power that equals to $30 \%$ of the control input power. Figure 3 shows the true evolution of all parameters (red line), together with the DEKF estimation (black line). In Figure 3f the DEKF's innovation is represented in red, whereas the (square root) of the innovation covariance is shown in black. The simulated human control input is shown in Figure 3g. For this simulation $r^{2}$ had a value of 0.7 , whereas $q^{2}$ was set to 0.4 .

It can be seen that the DEKF manages to estimate all the parameters quite closely, however with a very small bias for the neuromuscular frequency and neuromuscular damping ratio observed in Figure 3c and 3d, respectively. It can be seen that the innovation has zero mean and it falls within the square root of the innovation covariance matrix at each time step suggesting that the filter is optimal or close to optimal. The variance accounted for (VAF), ignoring the first 15 seconds is $99.87 \%$, meaning that the filter output explains $99.87 \%$ of the variance of the measured signal $u$. Note that the same variance accounted for can be obtained with different combinations of parameter estimation, that do not make physical sense in real life (a very high neuromuscular frequency, or a negative gain, for example), but can result from incorrect tuning of the DEKF.

\section{Time-varying parameters}

In order to test time-varying parameter identification, a similar simulation was performed, where the controlled dynamics and the equivalent human control model parameters changed according to sigmoid functions. The remnant had a break frequency of $3 \mathrm{rad} / \mathrm{s}$, and the gain was adjusted such that the remnant power at the control input location has a power that equals to $30 \%$ of the control input power. In reality, the remnant characteristics are also time varying, however in this simulation the remnant parameters were kept constant. At around second 40, the controlled dynamics $H_{c}$ start changing their break frequency from $3 \mathrm{rad} / \mathrm{s}$ towards $1 \mathrm{rad} / \mathrm{s}$. As a response, the simulated error position gain $K_{p}$ decreases, and the error velocity gain $K_{v}$ increases. Although in reality there isn't much change in the neuromuscular parameters and time delay, in this simulation $\omega_{n}$ decreased from $8 \mathrm{rad} / \mathrm{s}$ to $6 \mathrm{rad} / \mathrm{s}, \zeta_{n}$ increased from 0.3 to 0.5 and $\tau_{v}$ varied between 0.15 $\mathrm{s}$ and $0.25 \mathrm{~s}$ in a sine wave shap having a period of 90 seconds. These choices were made in order to test the parameter estimation behavior of the filter in a scenario where all parameters change. Figure 4 shows the true evolution of all parameters (red line), together with the DEKF estimation (black line). In Figure 4f the DEKF's innovation is shown in red, whereas the square root of the innovation covariance is shown in black. Simulated control is shown in Figure $4 \mathrm{~g}$. For this simulation, the same $r^{2}$ and $q^{2}$ factors were used as for the constant parameter simulation, having values of 0.7 and 0.4 , respectively.

The DEKF manages to converge to the true values although the initial guesses were not accurate for all the parameters. The DEKF estimates converge to the true values after about 15 seconds. The same bias is seen for the neuromuscular frequency and neuromuscular damping ratio. Similar to the constant parameter simulation, it can be seen that the innovation has zero mean and it falls within the square root of the innovation covariance matrix at each time step. The variance accounted for, ignoring the first 15 seconds, is $99.43 \%$ in this case.

Note that, since the remnant spectrum was kept constant during the simulation, $r^{2}$ and $q^{2}$ should in theory also be changed over time, to keep the same ratio of remnant power to control input and error signal variance. In reality however, the remnant spectrum changes with the controlled dynamics, and preliminary experiment data showed that the ratio between remnant power and control input power does not change

considerably, in this case. Therefore, constant values for $r^{2}$ and $q^{2}$ are assumed in the remainder of this paper. 


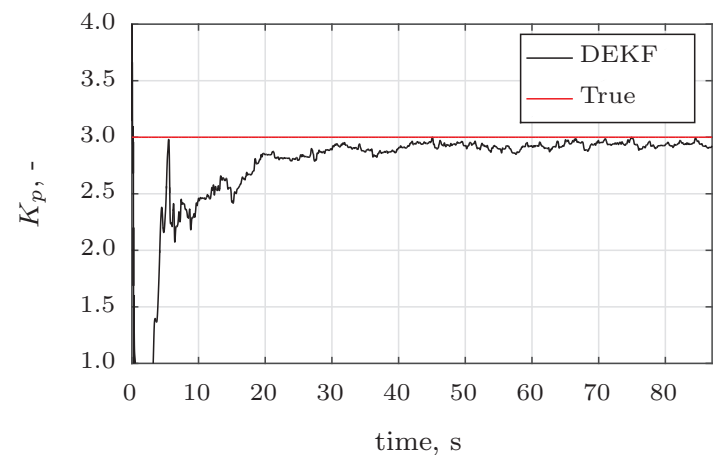

(a) Error position gain.

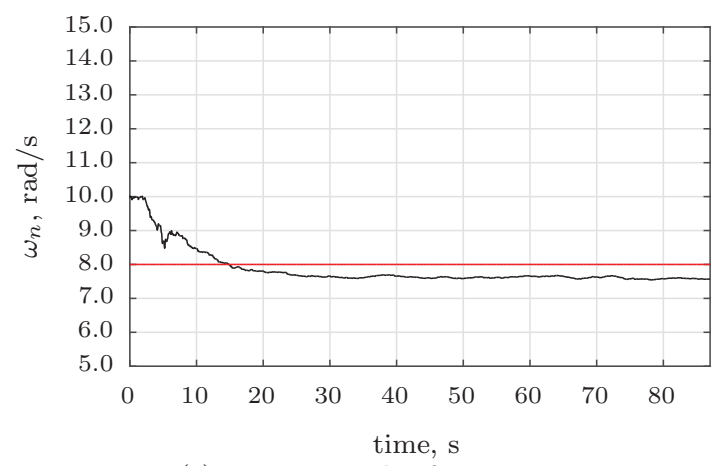

(c) Neuromuscular frequency.

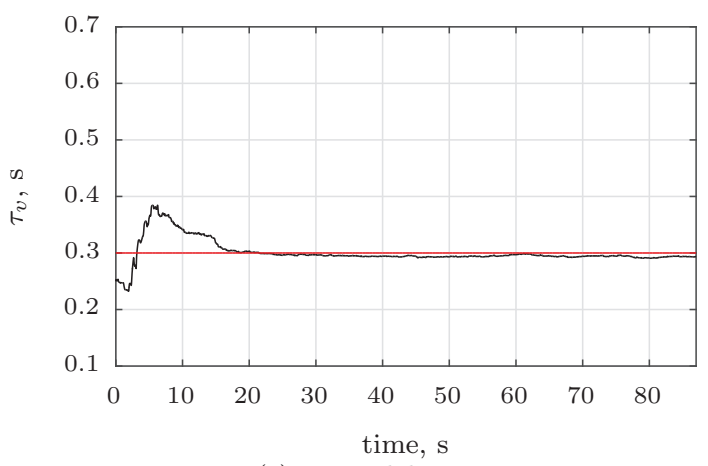

(e) Time delay.

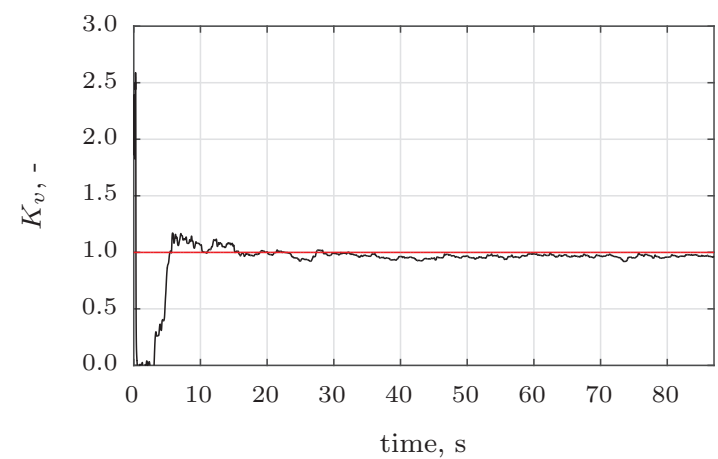

(b) Error velocity gain.

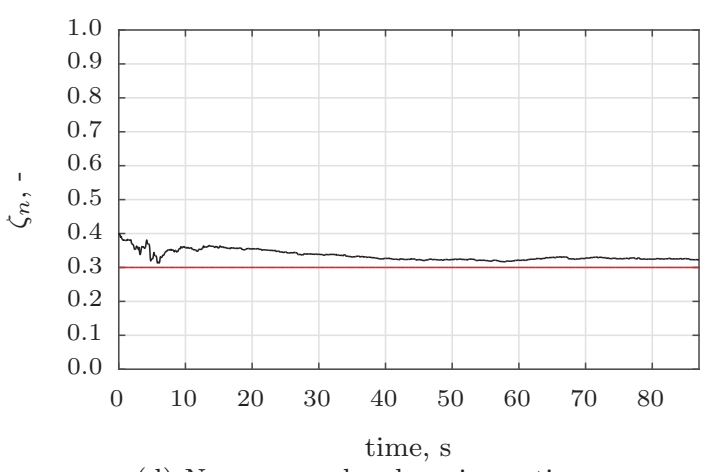

(d) Neuromuscular damping ratio.

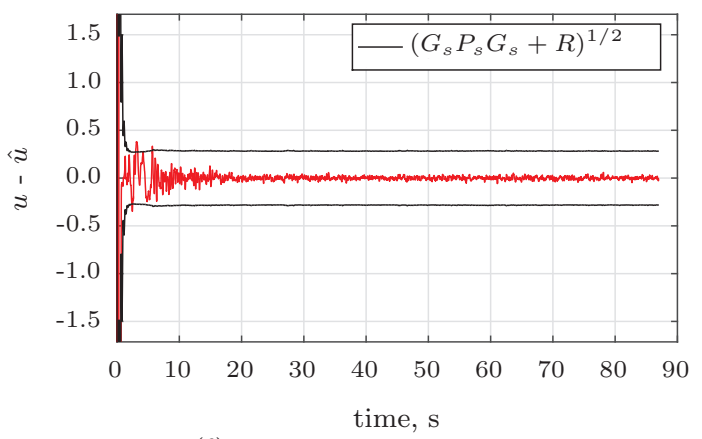

(f) Innovation covariance.

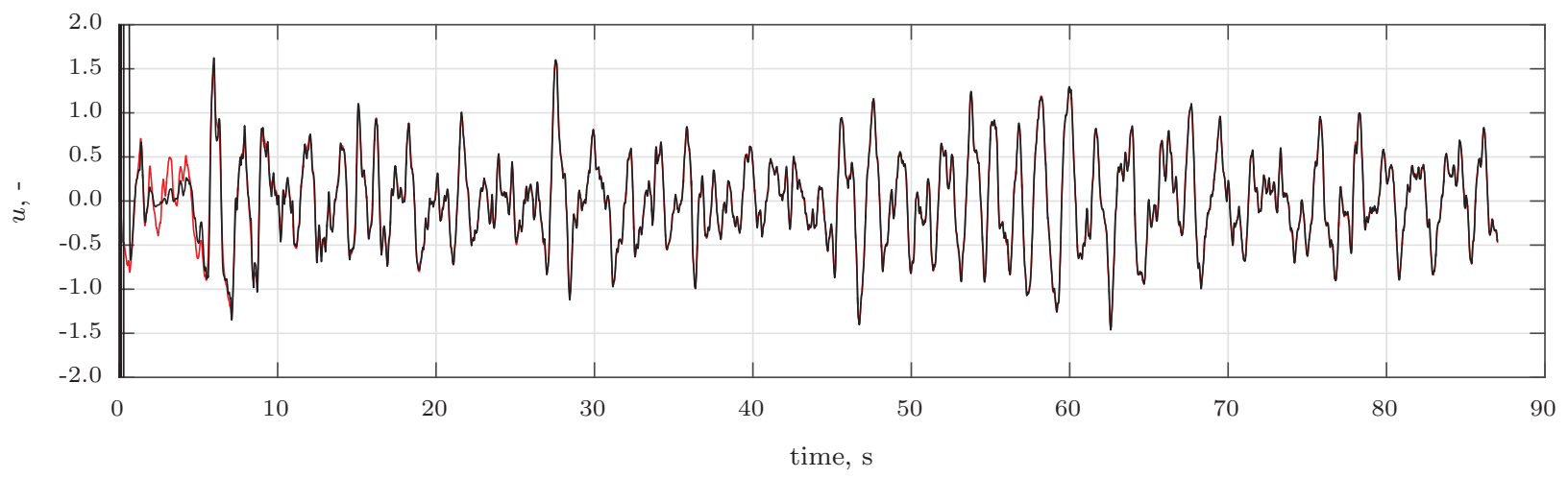

(g) Control input $u$.

Figure 3: Simulation DEKF results for constant parameters. 


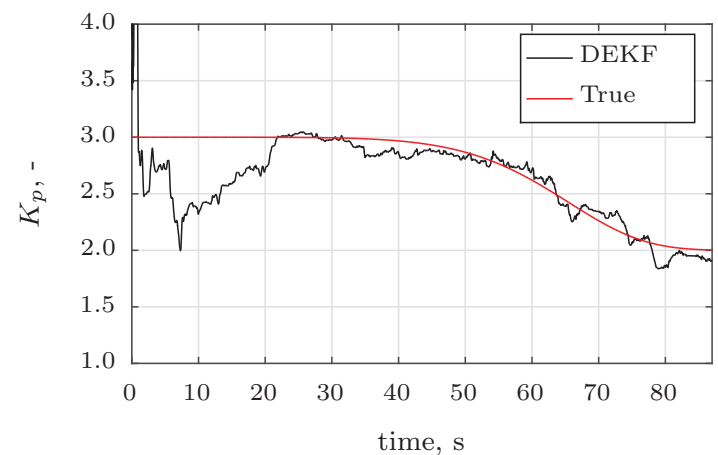

(a) Error position gain.

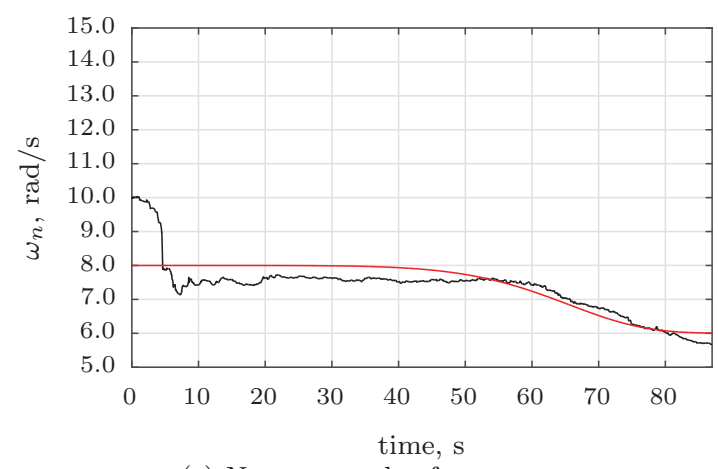

(c) Neuromuscular frequency.

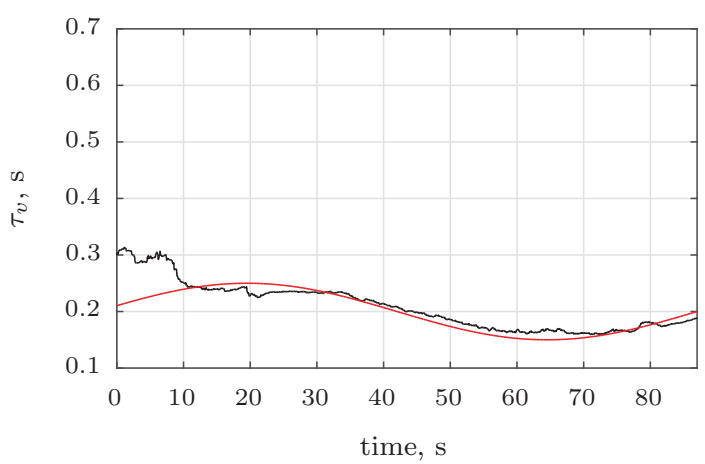

(e) Time delay.

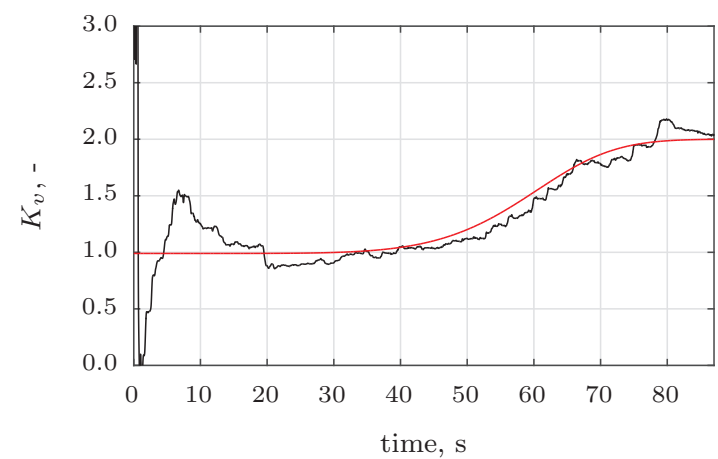

(b) Error velocity gain.

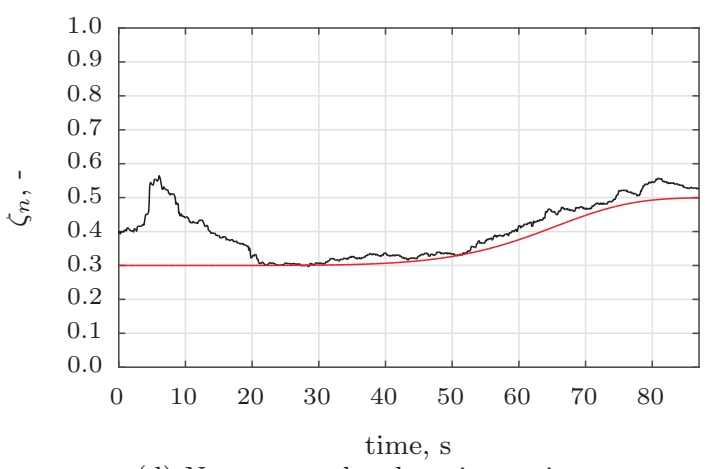

(d) Neuromuscular damping ratio.

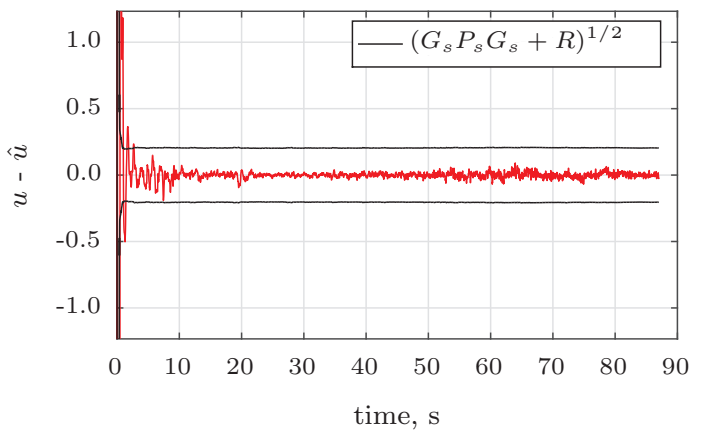

(f) Innovation (covariance).

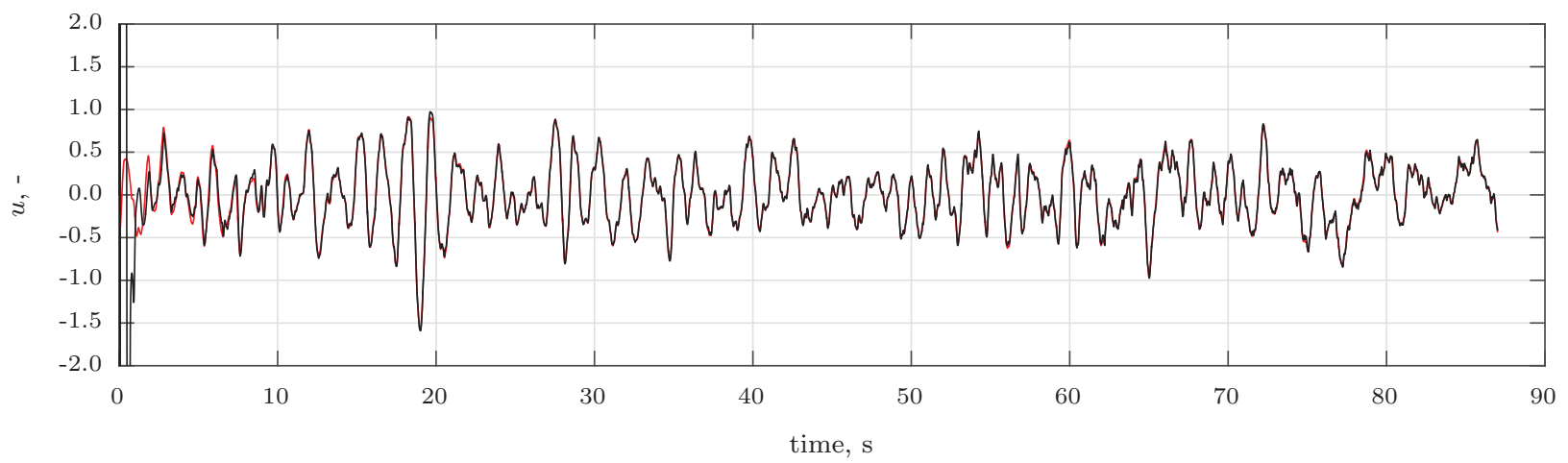

(g) Control input $u$.

Figure 4: Simulation DEKF results for time-varying parameters. 


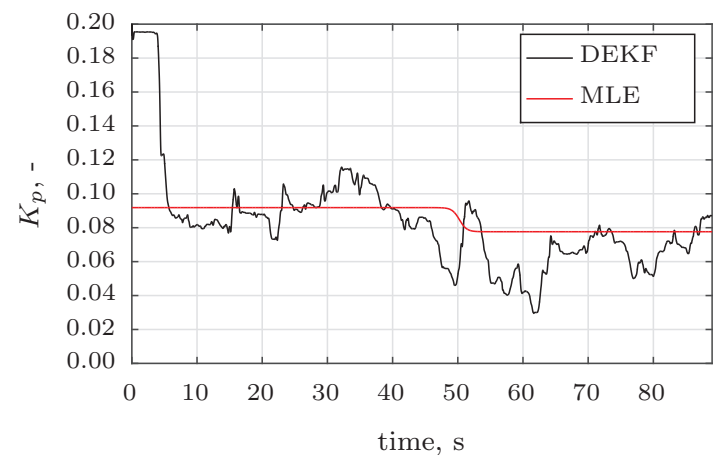

(a) Error position gain.

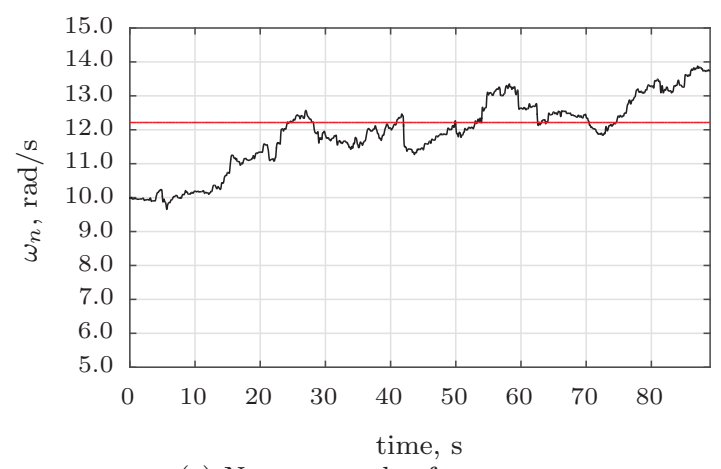

(c) Neuromuscular frequency.

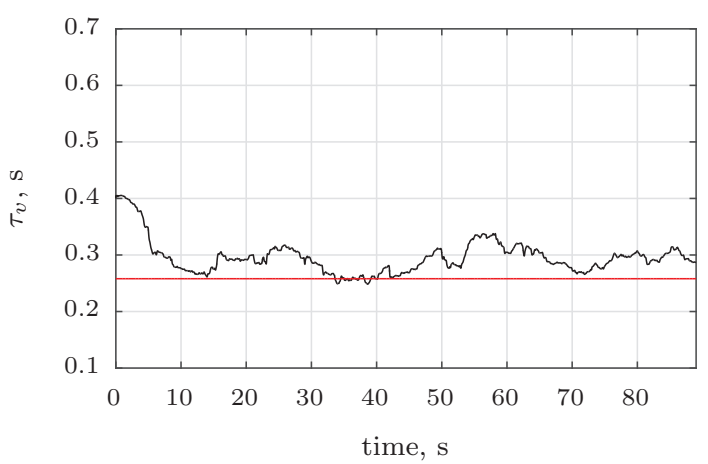

(e) Time delay.

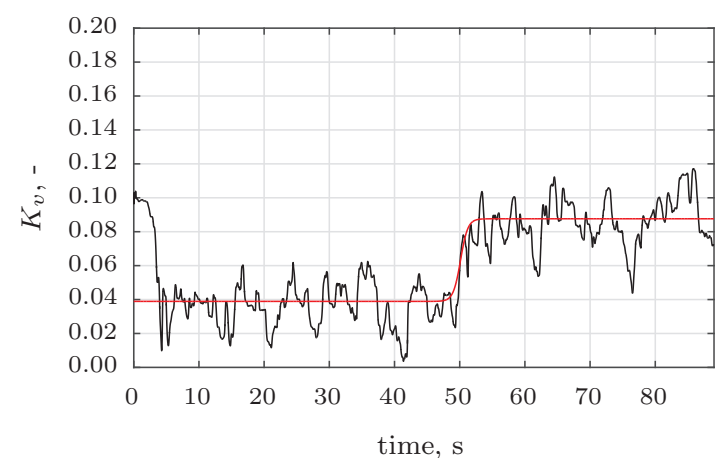

(b) Error velocity gain.

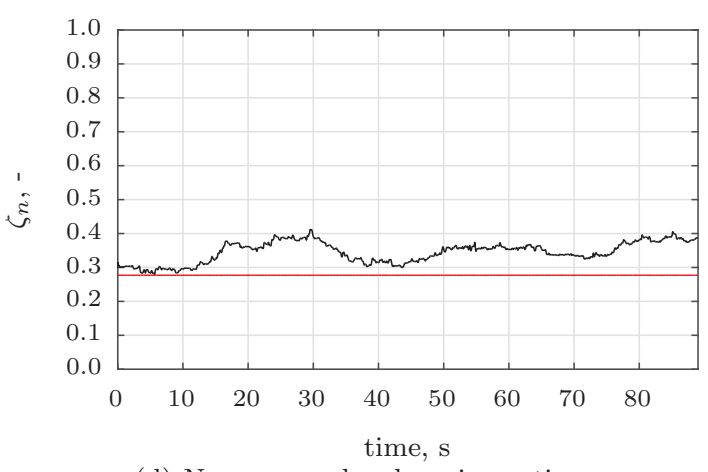

(d) Neuromuscular damping ratio.

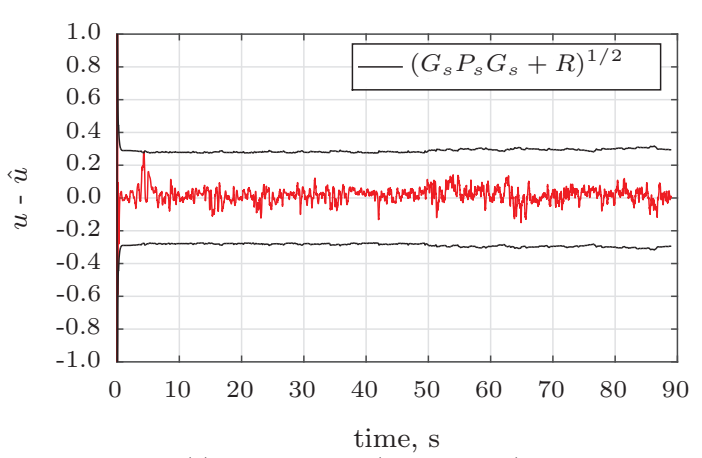

(f) Innovation (covariance).

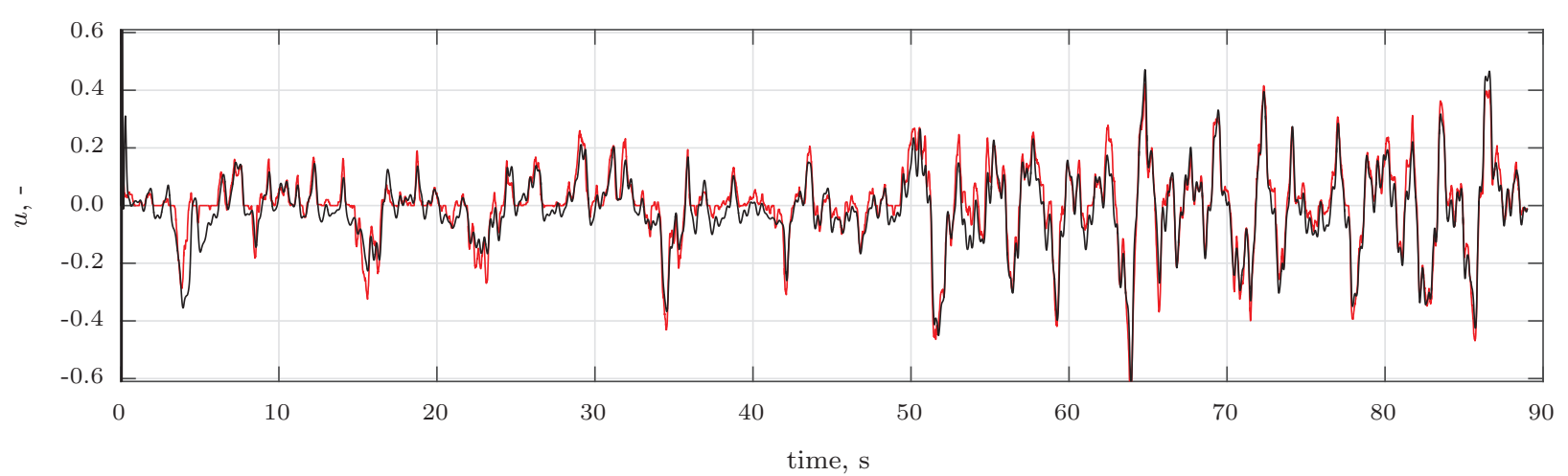

(g) Control input $u$.

Figure 5: DEKF compared to MLE on experimental data. 


\section{B. Time-varying multi-axis experiment}

Data from a time-varying multi-axis tracking task experiment performed in [5] was used to compare the performance of the DEKF to the MLE parameter estimation method. The experiment was aimed at identifying human adaption to changing controlled dynamics. In the mentioned experiment, the controlled dynamics changed from single integrator from double integrator following a sigmoid function. Therefore, the timevarying parameters of interest were the error position and error velocity gains, as well as two parameters describing the sigmoid function according to which the controlled dynamics change. Note that in this multiaxis experiment, the shape of the parameter change was required in order to properly identify human control parameters. Moreover, the neuromuscular parameters and the time delay were modeled as constants in the experiment.

From the multi-axis experiment, only pitch data from one subject was analyzed here, for comparison with our simulation. The data consists of the time average of the error signal $e$ and control input $u$ obtained from six, 90 second runs, in which the pitch dynamics changed from single to double integrator-like control. Figure 5 shows the results of the DEKF (in black lines), overlapped with the results from the MLE estimation method (in red lines). The initial parameter estimates of the DEKF were obtained using Equations (27) (31). $r^{2}$ was set to 4 , and $q^{2}$ was 0.4 , the same value as in the simulation data. The other initializations were the same as in the procedure described in Section IV]4.

Figure 5 shows the estimation performance of the DEKF compared to the MLE results from [5]. The parameter estimations are clearly similar for both methods, and the transition from single to double integrator dynamics is clear at around second 50. Since the DEKF does not assume a predefined scheduling for the parameter evolution, it has more variations, as it tries to estimate the parameters at each time step. The MLE method assumes constant time delay and neuromuscular parameters, and the DEKF results show that there is not much variation in these parameters caused by the change in controlled dynamics. A reason for the slow convergence of the neuromuscular frequency in Figure 5c is the bad initial estimate of neuromuscular frequency compared to the MLE estimate. However it can also be that the participant started the run with a lower neuromuscular frequency and kept increasing it during the run. The innovation of the DEKF has zero mean, and is well within the bounds of the innovation covariance matrix. The variance accounted for (calculated from second 20) is $86.59 \%$ for the MLE method, and $90.25 \%$ for the DEKF.

Note the large difference in the type of control input obtained from simulation data in Figure $4 \mathrm{~g}$ and the real control input of the participant of the multi-axis experiment, depicted in Figure $5 \mathrm{~g}$. The simulation control input is smooth, having no discontinuities, whereas the experiment control input does not seem as continuous, especially until around second 50. Note that the human control input in this case includes any possible nonlinearities in the joystick, such as noise and dead band. The fact that the DEKF still manages to estimate the parameters despite the relatively "poor" quality of the control input data from the experiment is promising for the applicability of the DEKF in real scenarios.

\section{Sensitivity to initial conditions}

Results from a few well-chosen tuning and initial parameter choices were presented in Section B. Since the state and parameter filters depend on each other's estimates at each time step, together with the fact that the state space equations are linearized through the use of the EKF, initial parameter estimates are critical for the convergence of the filter to meaningful parameters. The equations in Appendix $\mathrm{A}$ show that the equalization parameters $K_{p}$ and $K_{v}$ do not appear in the state equation (Equation (40)), but only in the measurement equation (Equation (41)). Thus, we expect that the DEKF will be less sensitive to the initial choice of these parameters. However, an initial guess that is too far from the true parameter in the state filter will affect the estimates of the parameter filter through the measurement update equation (Figure 2).

In this subsection, the DEKF is run on the same experimental data as in Figure 5 , but now with varying initial guesses for each of the five parameters. The time evolution of the respective parameter and the innovation are shown, where the divergence of the filter can be seen in some cases. The effect on the estimation of other parameters is not shown for brevity. The initial covariance matrix was increased depending on how far the initial guess was form the true value of the parameters, and the process noise covariance matrices were kept constant.

Figure [6] shows the results of the DEKF with four different levels of initial conditions for each parameter. In Figures 6a and 6c the error position gain and error velocity gains have initial conditions which are factors $0.1,1,2$ and 10 times the value of the true parameter, which we define as being the parameter value found 


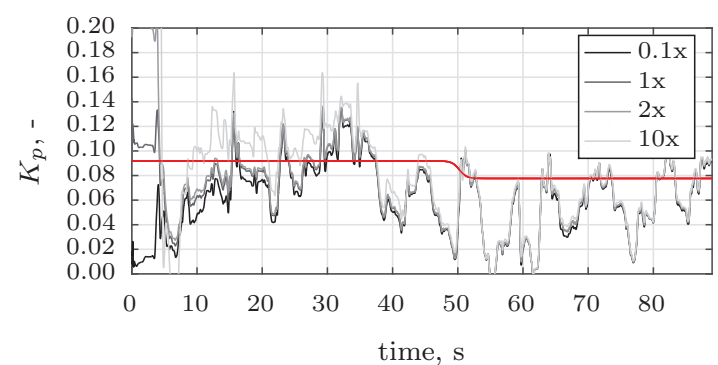

(a) Error position gain.

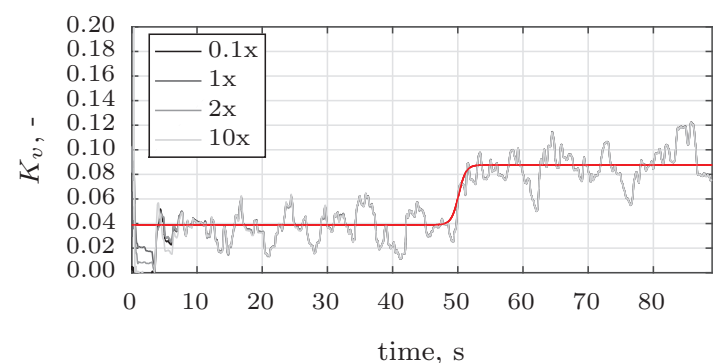

(c) Error velocity gain.

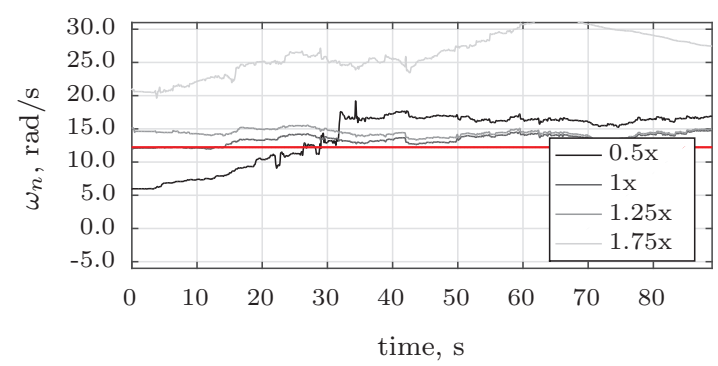

(e) Neuromuscular frequency.

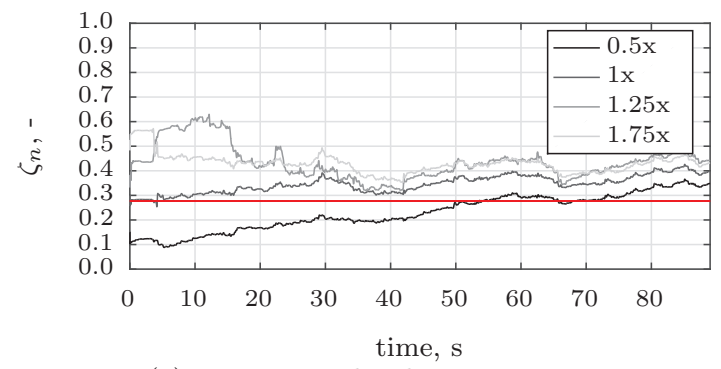

(g) Neuromuscular damping ratio.

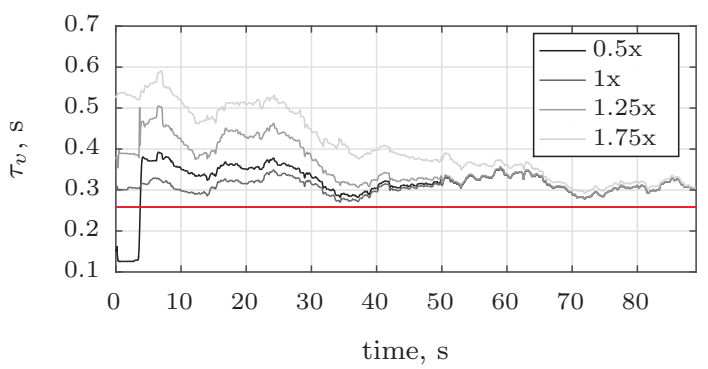

(i) Time delay,

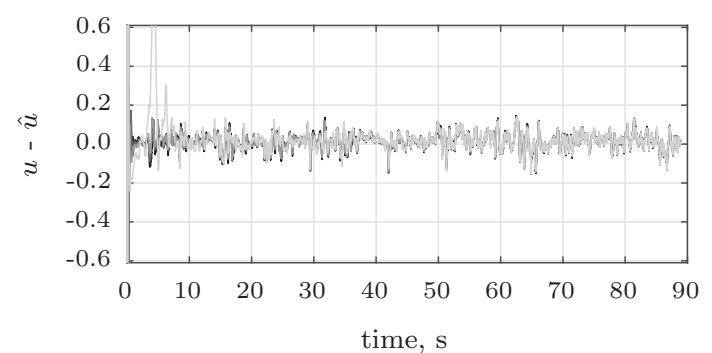

(b) Innovation.

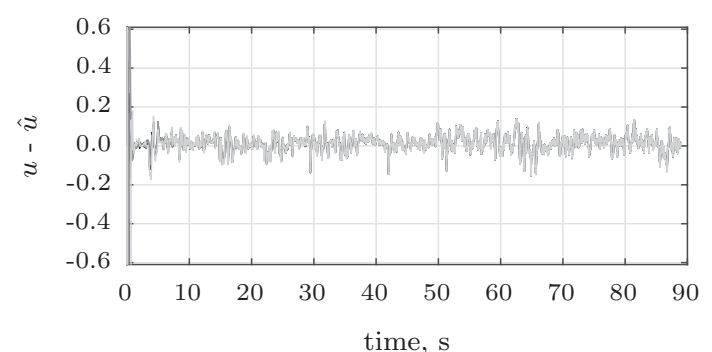

(d) Innovation.

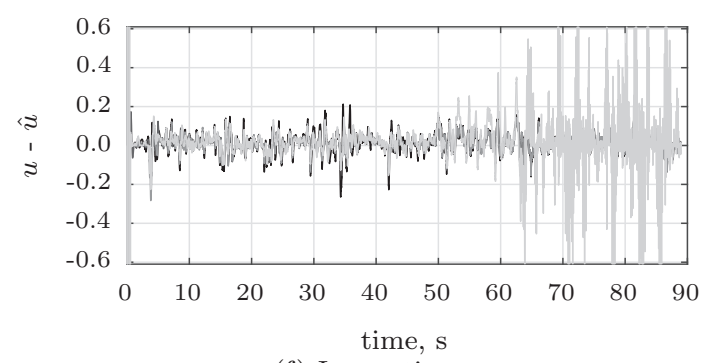

(f) Innovation.

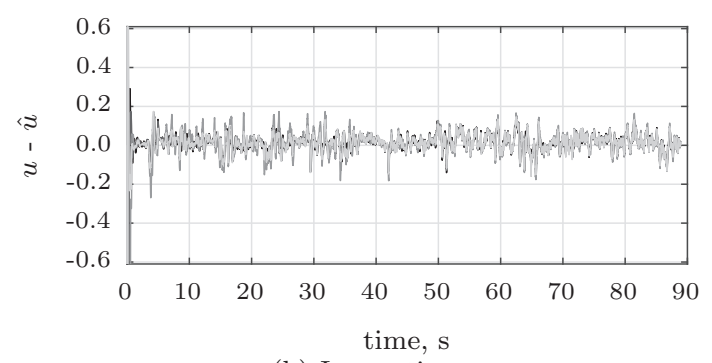

(h) Innovation.

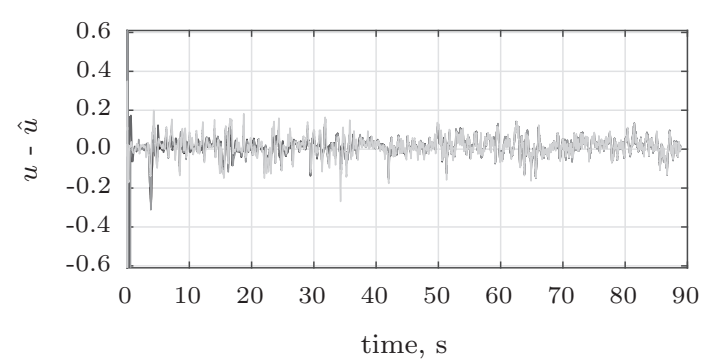

(j) Innovation.

Figure 6: Initial parameter estimate sensitivity. 
by the MLE estimation method. The filter manages to converge to the true parameter value in all cases. When $K_{p}$ starts with a value that is 10 times higher than the true value, the filter converges to the MLE estimate. However, at around second 5 , it has difficulty estimating the neuromuscular damping ratio and the time delay (not shown here). However, after second 10, all values are estimated correctly. For $\omega_{n}, \zeta_{n}$ and $\tau_{v}$, the initial conditions were chosen as the lower factors $0.5,1,1.25$ and 1.75, respectively, since the filter is more sensitive to these parameters. Figure 6e shows the estimation of the neuromuscular frequency. When the initial $\omega_{n}$ is 1 or 1.25 times the value of the true parameter, the filter converges for all parameters. The filter diverges when the initial guess is too high, and although it does not diverge for the 0.5 factor case, the estimate for the neuromuscular damping ratio is very high. The neuromuscular damping is shown in Figure $6 \mathrm{~g}$. The filter does not diverge for factors $1,1.25$ and 1.75 , however it seems to converge to slightly different final values. It also converges very slowly for a small initial guess of $\zeta_{n}$, but in this case the time delay is overestimated. Time delay estimation is shown is Figure 6i. where this parameter converges for all factors of $0.5,1,1.25$ and 1.75. For the highest initial condition, the neuromuscular damping ratio is underestimated. This is to be expected, as the time delay and the neuromuscular damping ratio both create a phase lag at higher frequencies and affect the output of the filter in a similar way. Therefore, a higher estimated time delay is compensated by a lower neuromuscular damping.

\section{Discussion}

The goal of this study was to develop a method for estimating time-varying human control behavior based on a Dual Extended Kalman Filter. A challenge using this approach is the inclusion of process and measurement noise, which makes it a particularly difficult task when the system is represented by a human in a closed-loop task. In this implementation, the remnant was assumed to be a combination of a zero-mean, Gaussian white noise, included as both process and measurement noise having powers proportional to the input and the output of the human control model. Although Levison et al. found that the remnant has colored characteristics $\frac{16}{16}$ more accurate modeling and inclusion of the remnant spectrum in the state space is subject to future work. Simulations were used to assess the performance of the DEKF implementation and to provide insights on how to tune the filter for optimal performance. Constant and time-varying parameters were used, and it was shown that the filter could estimate the parameters well in both cases, although with a small bias for the neuromuscular parameters as shown in Figure $4 \mathrm{c}$ and Figure 4d, The simulations resulted in some practical guidelines on how to obtain initial parameter estimates, and how to obtain the process and measurement noise covariance matrices. The filter was then applied on data obtained from a time-varying multi-axis control experiment,, 5 with the same tuning obtained from the simulation data. The only parameter that needed to be adjusted was the measurement noise covariance matrix, which had to be increased in the case of the experimental data. This is not surprising, since in reality there are more noise sources present than in the simulations. For example, the control input device that was used in the experiment could add additional noise in the system, which has to be accounted for by the filter. Another interesting fact is that the control input in the experiment, shown in Figure $5 \mathrm{~g}$, was smooth than the ones from the simulation, shown in Figures $3 \mathrm{~g}$ and $4 \mathrm{~g}$. However, the DEKF produced parameter estimates that are very close to the ones obtained using the Maximum Likelihood Estimation method in [5].

Due to the interdependence of the the state and parameter filters, and the linearization performed by the Extended version of the Kalman filter, a trade-off has to be made in how fast the variation of the parameters is captured. Increasing the process noise of the parameters could capture faster changes, however there is a risk that the filter diverges if one estimate is too far from the true value, which will in turn affect the estimation of all parameters. Having a lower value for the parameter process noise will capture changes slower, however with a lower risk of filter divergence, especially since the changes in human manual control parameters are generally small.

The sensitivity of our approach to the initial choice of parameter estimates was investigated in Section C where it was shown that the DEKF is more sensitive to the choice of initial neuromuscular parameters and time delay than the initial choice of equalization parameters. However, these parameters do not typically have much variation during human control, even with changes in vehicle dynamics. Therefore, generic values can be used during filter initialization, as shown in Equations (29) - (31). The sensitivity analysis also showed that the filter can have the same output error even though the estimated parameters are quite different. For example, when the time delay was estimated as being too low, the filter overestimated the neuromuscular damping ratio, while the filter output was identical. The filter managed to converge to the true value 
only after a long time, after a few tens of seconds. This shows that even if the filter does not diverge, it happens that the estimated parameters do no make physical sense, and an initial parameter estimate will make the filter convergence slow. Furthermore, if the system is not sufficiently excited, the estimation of the parameters also becomes problematic because of observability issues.

There are a few advantages using the DEKF. First, the state-space has a low dimension, the state filter having 7 states and the parameter filter 3 states, which makes the filter computationally fast. Second, it can estimate changes in human manual control behavior without having a priori knowledge on how the parameters change over time. Third, it can be applied to experiment data directly, without the need of averaging the time signals in advance, technique required in previous research in order to increase the signalto-noise ratio ${ }^{4}$ Lastly, initial parameters estimates can be obtained using some simple guidelines, and the only parameter that needs to be tuned when going from simulation to experiment data was $r^{2}$, the control input measurement noise factor. Note that the tuning was tailored to a compensatory tracking task, meaning that when applied to a different type of human control task, different tuning of the filter might be required.

As future work, the colored characteristics of the human remnant will be included in the state space representation explicitly. Furthermore, the possibility of automatically choosing the factors $r^{2}$ and $q^{2}$ will be considered. In order to increase the applicability of the filter, its use for the multi-channel human control models will be investigated. Lastly, a Dual Unscented Kalman Filter approach will be investigated in order to analyze its performance in terms of speed compared to the DEKF. The ultimate goal of this research is the development of a hardware solution that can achieve real-time identification of human manual control, which could be used for human state monitoring, as well as adaptive haptic interfaces.

\section{Conclusions}

A Dual Extended Kalman Filter was implemented for the identification of time-varying human manual control behavior. The state space representation of human manual control was split into two filters that run concurrently, a state filter that estimates the equalization dynamics, and a parameter that estimates human operator neuromuscular parameters and the time delay, which was included in the state space as a Padé approximation. All human manual control parameters were modeled as having constant dynamics and driven by a white noise process ("random walk").

The DEKF manages to estimate time-varying human control behavior in both simulated and experimental data. Simple guidelines are proposed for the tuning of the process and measurement covariance matrices and the initial parameter estimates. The tuning was performed on simulation data, and when applied on the experiment data, only an increase in measurement process noise power was required in order for the filter to converge. Poor initial choices of parameters, especially for the neuromuscular parameters and the time delay, can lead to filter divergence or parameter estimates that do not have a real physical interpretation. Another limitation of the filter is the need for long convergence time (a few tens of seconds), if the initial parameter estimates are far from the true values.

Since the scheduling of a parameter change is not required in advance, and the filter shows promising results when applied to experiment data without the need of time-averaging of multiple runs, the use of DEKF represents a viable option for the real-time identification of human control in manual tracking tasks. 


\section{A. Human controller state space representation}

The transfer function $H_{p}$ was transformed into its state space controllable canonical form in order to be implemented in the Kalman filter. The time delay $\tau_{v}$ was approximated by a $3^{\text {rd }}$ order Padé filter. The main reason for the choice of the $3^{\text {rd }}$ order approximation is the fact that the Extended Kalman filter uses a first-order linearization of the system. Therefore, a higher order in the Padé approximation would result in the Extended Kalman filter to not converge due to the high non-linearities in the state space.

In this section, the full form of the state space model, together with the total derivate of $G_{p, k}^{\text {tot }}$ are presented.

$$
\begin{aligned}
& \dot{\boldsymbol{x}}_{\boldsymbol{s}}(t)=f\left(\boldsymbol{x}_{\boldsymbol{s}}(t), e(t), \boldsymbol{\theta}(t)\right)+\boldsymbol{w}_{\boldsymbol{s}}(t) \\
& \dot{\boldsymbol{\theta}}(t)=\boldsymbol{w}_{\boldsymbol{p}}(t) \\
& u(t)=g\left(\boldsymbol{x}_{\boldsymbol{s}}(t), \boldsymbol{\theta}(t)\right)+v(t) \\
& \boldsymbol{x}_{\boldsymbol{s}}=\left[\begin{array}{lllllll}
x_{s, 1} & x_{s, 2} & x_{s, 3} & x_{s, 4} & x_{s, 5} & K_{p} & K_{v}
\end{array}\right]^{T} \\
& \boldsymbol{\theta}=\left[\begin{array}{lll}
\omega_{n} & \zeta_{n} & \tau_{v}
\end{array}\right]^{T} \\
& f\left(\boldsymbol{x}_{\boldsymbol{s}}, e, \boldsymbol{\theta}\right)=\left[\begin{array}{c}
x_{s, 2} \\
x_{s, 3} \\
x_{s, 4} \\
x_{s, 5} \\
e-x_{s, 3}\left(12 \omega_{n}^{2} \tau_{v}^{2}+120 \zeta_{n} \omega_{n} \tau_{v}+120\right) / \tau_{v}^{3}-x_{s, 2}\left(60 \tau_{v} \omega_{n}^{2}+240 \zeta_{n} \omega_{n}\right) / \tau_{v}^{3}- \\
\cdots-x_{s, 4}\left(\omega_{n}^{2} \tau_{v}^{3}+24 \zeta_{n} \omega_{n} \tau_{v}^{2}+60 \tau_{v}\right) / \tau_{v}^{3}-x_{s, 5}\left(2 \zeta_{n} \omega_{n} \tau_{v}^{3}+12 \tau_{v}^{2}\right) / \tau_{v}^{3}-120 x_{s, 1} \omega_{n}^{2} / \tau_{v}^{3} \\
0 \\
0
\end{array}\right] \\
& g\left(\boldsymbol{x}_{\boldsymbol{s}}, \boldsymbol{\theta}\right)=\left[\begin{array}{c}
x_{s, 3}\left(12 K_{p} \omega_{n}^{2} \tau_{v}^{2}-60 K_{v} \omega_{n}^{2} \tau_{v}\right) / \tau_{v}^{3}-K_{v} \omega_{n}^{2} x_{s, 5}+x_{s, 2}\left(120 K_{v} \omega_{n}^{2}-60 K_{p} \omega_{n}^{2} \tau_{v}\right) / \tau_{v}^{3}- \\
\cdots-x_{s, 4}\left(K_{p} \omega_{n}^{2} \tau_{v}^{3}-12 K_{v} \omega_{n}^{2} \tau_{v}^{2}\right) / \tau_{v}^{3}+120 K_{p} \omega_{n}^{2} x_{s, 1} / \tau_{v}^{3}
\end{array}\right] \\
& G_{p, k}^{t o t}=\left.\frac{d g\left(\boldsymbol{x}_{s, k}^{-}, \boldsymbol{\theta}\right)}{d \boldsymbol{\theta}}\right|_{\boldsymbol{\theta}=\boldsymbol{\theta}_{k}^{-}} \\
& \frac{d g\left(\boldsymbol{x}_{s, k}^{-}, \boldsymbol{\theta}\right)}{d \boldsymbol{\theta}}=\frac{\partial g\left(\boldsymbol{x}_{s, k}^{-}, \boldsymbol{\theta}\right)}{\partial \boldsymbol{\theta}}+\frac{\partial g\left(\boldsymbol{x}_{s, k}^{-}, \boldsymbol{\theta}\right)}{\partial \boldsymbol{x}_{s, k}^{-}} \frac{d \boldsymbol{x}_{s, k}^{-}}{d \boldsymbol{\theta}} \\
& \frac{d \boldsymbol{x}_{s, k}^{-}}{d \boldsymbol{\theta}}=\frac{\partial f\left(\boldsymbol{x}_{s, k-1}^{+}, e_{k-1}, \boldsymbol{\theta}\right)}{\partial \boldsymbol{\theta}}+\frac{\partial f\left(\boldsymbol{x}_{s, k-1}^{+}, e_{k-1}, \boldsymbol{\theta}\right)}{\partial \boldsymbol{x}_{s, k-1}^{+}} \frac{d \boldsymbol{x}_{s, k-1}^{+}}{d \boldsymbol{\theta}} \\
& \frac{d \boldsymbol{x}_{s, k-1}^{+}}{d \boldsymbol{\theta}}=\frac{d \boldsymbol{x}_{s, k-1}^{-}}{d \boldsymbol{\theta}}-K_{s, k-1} \frac{d g\left(\boldsymbol{x}_{s, k-1}^{-}, \boldsymbol{\theta}\right)}{d \boldsymbol{\theta}}
\end{aligned}
$$

where the Equations (42) - (45) show how the complete derivative of the states of the parameter filter with the respect to the output equation is calculated. Note that the values of $K_{s, k-1}, \frac{d g\left(\boldsymbol{x}_{s, k-1}^{-}, \boldsymbol{\theta}\right)}{d \boldsymbol{\theta}}$ and $\frac{d \boldsymbol{x}_{s, k-1}^{-}}{d \boldsymbol{\theta}}$ from the previous time step are required, in order to compute the derivative numerically at the current time step. The calculation of the complete derivative is necessary since the states of the parameter filter do not

directly appear in the output equation $g$. The initial values for $K_{s, k-1}, \frac{d g\left(\boldsymbol{x}_{s, k-1}^{-}, \boldsymbol{\theta}\right)}{d \boldsymbol{\theta}}$ and $\frac{d \boldsymbol{x}_{s, k-1}^{-}}{d \boldsymbol{\theta}}$ are initialized with 0 at the beginning of the estimation. 


\section{References}

${ }^{1}$ McRuer, D. T. and Jex, H. R., "A review of quasi-linear pilot models," Human Factors in Electronics, IEEE Transactions on, , No. 3, 1967, pp. 231-249.

${ }^{2}$ van Paassen, M. M. and Mulder, M., "Identification of Human Operator Control Behaviour in Multiple-Loop Tracking Tasks," Proceedings of the Seventh IFAC/IFIP/IFORS/IEA Symposium on Analysis, Design and Evaluation of Man-Machine Systems, Kyoto Japan, Pergamon, Kidlington, 16-18 Sept. 1998, pp. 515-520.

${ }^{3}$ Nieuwenhuizen, F. M., Zaal, P. M., Mulder, M., Van Paassen, M. M., and Mulder, J. A., "Modeling Human Multichannel Perception and Control using Linear Time-invariant Models," Journal of Guidance, Control, and Dynamics, Vol. 31, No. 4, 2008, pp. 999-1013.

${ }^{4}$ Zaal, P. M. T., Pool, D. M., Chu, Q. P., van Paassen, M. M., Mulder, M., and Mulder, J. A., "Modeling Human Multimodal Perception and Control Using Genetic Maximum Likelihood Estimation," Journal of Guidance, Control, and Dynamics, Vol. 32, No. 4, July-Aug. 2009, pp. 1089-1099.

${ }^{5}$ Zaal, P. M., "Manual Control Adaptation to Changing Vehicle Dynamics in Roll-Pitch Control Tasks," Journal of Guidance, Control, and Dynamics, 2016, pp. 1046-1058.

${ }^{6}$ Zaal, P. and Sweet, B., "Estimation of time-varying pilot model parameters," AIAA Modeling and Simulation Technologies Conference, 2011, p. 6474 .

${ }^{7}$ Kalman, R. E. et al., "Contributions to the theory of optimal control," Bol. Soc. Mat. Mexicana, Vol. 5, No. 2, 1960, pp. 102-119.

${ }^{8}$ Goodwin, G. C. and Sin, K. S., Adaptive filtering prediction and control, Courier Corporation, 2014.

${ }^{9}$ Nelson, L. and Stear, E., "The simultaneous on-line estimation of parameters and states in linear systems," IEEE Transactions on automatic Control, Vol. 21, No. 1, 1976, pp. 94-98.

${ }^{10}$ Wan, E. A. and Nelson, A. T., "Dual Kalman filtering methods for nonlinear prediction, smoothing, and estimation," Advances in neural information processing systems, Vol. 9, 1997.

${ }^{11}$ Walder, G., Campestrini, C., Lienkamp, M., and Jossen, A., "Adaptive State and Parameter Estimation of Lithium-Ion Batteries Based on a Dual Linear Kalman Filter," The Second International Conference on Technological Advances in Electrical, Electronics and Computer Engineering (TAEECE2014), The Society of Digital Information and Wireless Communication, 2014, pp. $16-24$.

${ }^{12}$ Wenzel, T. A., Burnham, K., Blundell, M., and Williams, R., "Dual extended Kalman filter for vehicle state and parameter estimation," Vehicle System Dynamics, Vol. 44, No. 2, 2006, pp. 153-171.

${ }^{13}$ Schiess, J. R. and Roland, V. R., "Kalman filter estimation of human pilot-model parameters," 1975.

${ }^{14}$ Levison, W. H., Elkind, J. I., and Ward, J. L., Studies of multivariable manual control systems: A model for task interference, National Aeronautics and Space Administration, 1971.

${ }^{15}$ Friedland, B., "Treatment of bias in recursive filtering," IEEE Transactions on Automatic Control, Vol. 14, No. 4, 1969, pp. 359-367.

${ }^{16}$ Levison, W., Baron, S., and Kleinman, D., "A Model for Human Controller Remnant," Man-Machine Systems, IEEE Transactions on, Vol. 10, No. 4, Dec 1969, pp. 101-108.

${ }^{17}$ Jazwinski, A. H., Stochastic processes and filtering theory, Courier Corporation, 2007.

${ }^{18}$ Vachhani, P., Narasimhan, S., and Rengaswamy, R., "Recursive state estimation in nonlinear processes," American Control Conference, 2004. Proceedings of the 2004, Vol. 1, IEEE, 2004, pp. 200-204. 